\title{
On Bandlimited Signals with Minimal Space/Time-Bandwidth Product
}

\author{
Y. V. Venkatesh, S. Kumar Raja and G. Vidya Sagar \\ Department of Electrical Engineering \\ Indian Institute of Science \\ Bangalore 560012, INDIA
}

\begin{abstract}
We deal with the problem of determining bandlimited signals which have the lowest space/time-bandwidth product. We propose two approaches to the problem: the first is based on a modification of the Gaussian function; and the second, on the solution of a set of nonlinear algebraic equation obtained from the samples of the signal.
\end{abstract}

\section{INTRODUCTION}

We deal with real signals, $f \in L_{2}$, which are treated as functions of the real variable $x \in \mathbb{R}$ (i.e., the class of square integrable functions) having unit energy and centered at the origin. The independent variable $x$ can denote either time (for dealing with time-dependent phenomena) or space (for describing space-dependent functions, like scanlines of images). In what follows, we use the terms 'space' and 'time' interchangeably.

Let $F(\omega)$ denote the Fourier transform of $f(x)$. Based on the assumptions made above, we can establish the following identities:

$$
\int_{-\infty}^{\infty}|f(x)|^{2} \mathrm{~d} x=1=\frac{1}{2 \pi} \int_{-\infty}^{\infty}|F(\omega)|^{2} \mathrm{~d} \omega,
$$

It is the practice to denote the space localization of a signal by its "effective spatial-width", $\left(\Delta_{x}\right)$ :

$$
\left(\Delta_{x}\right)^{2}=\int_{-\infty}^{\infty} x^{2} f^{2}(x) \mathrm{d} x
$$

Similarly, the frequency localization of a signal is described by its "effective spectral-width":

$$
\left(\Delta_{\omega}\right)^{2}=\frac{1}{2 \pi} \int_{-\infty}^{\infty} \omega^{2}|F(\omega)|^{2} \mathrm{~d} \omega
$$

It is well known that $f(x)$ and $F(\omega)$ cannot both be of short duration. And this is made explicit (a) qualitatively by the scaling theorem,

$$
a f(a x) \Leftrightarrow F\left(\frac{\omega}{a}\right)
$$

and (b) quantitatively by the uncertainty principle which places a lower bound on the product of effective spatial and spectral widths of continuous signals (also called the spacebandwidth product (SBP)) [1]:

$$
\left(\Delta_{x}\right)^{2}\left(\Delta_{\nu}\right)^{2} \geq \frac{1}{4}
$$

The equality in (4) is satisfied only by the Gaussian function, $\exp \left(-\frac{x^{2}}{2}\right)$. (For a survey on the uncertainty inequality (4), see [2].)

Let $\mathcal{B}$ denote the class of continuous and finite energy signals which are bandlimited, i.e.,

$$
|F(\omega)|=0, \quad \forall|\omega|>W
$$

For signals belonging to $\mathcal{B}$, Ishii et al. [3], [4] obtain a strict uncertainty inequality based on the further assumption that $F(\omega)=0$ for $|\omega|=W$. However, the following problem is still open:

Problem 1: What is the function belonging to $\mathcal{B}$ which attains the lowest uncertainty product? It appears that an answer to this problem does not exist. In this paper, we provide an answer to a slightly modified problem:

Problem 2: How close can the uncertainty product of functions belonging to $\mathcal{B}$ get to the lower bound obtained in (4)?

The rest of the paper is organized as follows. In Sec.II , we present the first approach to Problem 2, based on a modification of the Gaussian function. In Sec.III, we propose a general approach to the same problem using our earlier results [5] on the sampled signal. We conclude the paper in Sec. IV.

\section{MAin RESUlts: SOlution 1}

Using (2) and the properties of Fourier transform, it can be shown that the effective spatial width is finite only if $\left|\frac{d F(\omega)}{d \omega}\right|$ is square integrable. Therefore, for $f \in \mathcal{B}$, we require that $F(\omega)$ have finite derivatives at $\omega=W$ and $\omega=-W$. (Similarly, the effective spectral width is finite only if $\left|\frac{d f(x)}{d x}\right|$ is square integrable.)

Let

$$
F(\omega)=G(\omega) H(\omega)
$$

where

$$
G(\omega)=C \exp \left(\frac{-\omega^{2}}{2 \sigma^{2}}\right)
$$


and

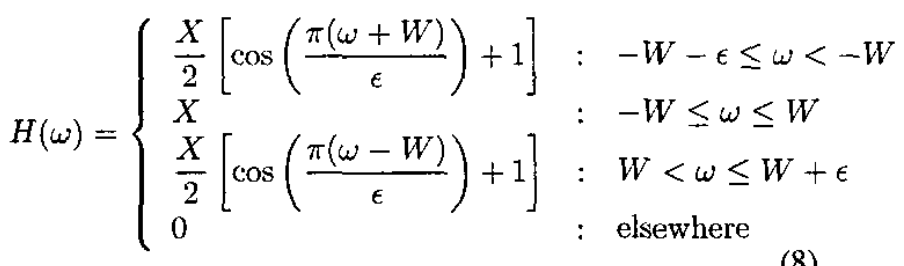

\begin{tabular}{|c|c|c|}
\hline Sl.No & $\sigma$ & Product \\
\hline 1 & 2.0 & 1.19870 \\
\hline 2 & 1.8 & 0.74649 \\
\hline 3 & 1.7 & 0.57802 \\
\hline 4 & 1.5 & 0.36008 \\
\hline 5 & 1.3 & 0.27172 \\
\hline 6 & 1.0 & 0.25026 \\
\hline 7 & 0.9 & 0.25002 \\
\hline 8 & 0.8 & 0.25000 \\
\hline 9 & 0.7 & 0.25000 \\
\hline 10 & 0.5 & 0.25000 \\
\hline
\end{tabular}

TABLE I

Fourier Transform of the interpolating function

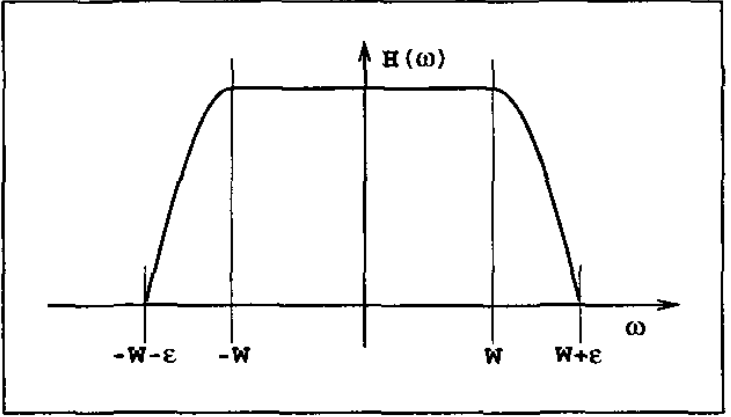

Fig. 1. Fourier transform of the interpolating function

where $X=1.0$, and $\epsilon>0$ is a small positive quantity. See Fig. 1 for the plot of $H(\omega)$ which satisfies the following conditions:

$$
\begin{gathered}
H(W)=X, H(W+\epsilon)=0, \\
H(-W)=X, H(-W-\epsilon)=0 \\
\left.\frac{\mathrm{d} H(\omega)}{\mathrm{d} \omega}\right|_{W+\epsilon}=0,\left.\frac{\mathrm{d} H(\omega)}{\mathrm{d} \omega}\right|_{W}=0, \\
\left.\frac{\mathrm{d} H(\omega)}{\mathrm{d} \omega}\right|_{-W-\epsilon}=0,\left.\frac{\mathrm{d} H(\omega)}{\mathrm{d} \omega}\right|_{-W}=0
\end{gathered}
$$

¿From (8) and (6), we can conclude that $F(\omega)$ is a bandlimited function with bandlimit $W+\epsilon$. Also $F(\omega)=0, \forall|\omega|=$ $W+\epsilon$, and is differentiable everywhere. In the next section, we evaluate (2) and (3) for any given function $f$.

See Tables 1 and 2 for the (squared) SBP of $F(\omega)$ for various of values $\sigma$ (in $G(\omega)$ ). The important point to be noted is that for any small value of $\epsilon>0$, there are bandlimited functions of the type $F(\omega)$ whose uncertainty product is very close to the lower bound in (4). More precisely, there is a sequence of bandlimited functions whose (squared) $S B P$ approaches the lower bound in (4) for a given bandwidth $W$ and $\epsilon$.

In the next section, we propose a new approach (based on our earlier results [5]) to find the optimal bandlimited signal whose SBP is the lowest.

\section{Main Results: Solution 2}

We now exploit the fact that a bandlimited signal is completely represented by a discrete set of signal samples if the
CONVERGENCE OF UNCERTAINTY PRODUCT USING COSINE INTERPOLATION FUNCTION WITH $\epsilon=0.1$ AND $W=\pi$

\begin{tabular}{|c|c|c|}
\hline SI.No & $\sigma$ & Product \\
\hline 1 & 2.0 & 10.42400 \\
\hline 2 & 1.8 & 5.66300 \\
\hline 3 & 1.7 & 3.86440 \\
\hline 4 & 1.5 & 1.49740 \\
\hline 5 & 1.3 & 0.50648 \\
\hline 6 & 1.0 & 0.25349 \\
\hline 7 & 0.9 & 0.25031 \\
\hline 8 & 0.8 & 0.25001 \\
\hline 9 & 0.7 & 0.25000 \\
\hline 10 & 0.5 & 0.25000 \\
\hline
\end{tabular}

TABLE II

CONVERGENCE OF UNCERTAINTY PRODUCT USING COSINE INTERPOLATION FUNCTION WITH $\epsilon=0.01$ AND $W=\pi$

sampling is done at or above the Nyquist rate. To this end, let $\{f[n]\}_{n \in \mathbb{Z}}$ denote the samples obtained by sampling uniformly (with the sampling interval $=X$ ) $a$ bandlimited signal $f(x)$. In [5], we have derived an expression for the SBP of $f(x)$ in terms of its samples.

The expressions for the squares of effective spectral and spatial widths, $\Delta_{\omega}$ and $\Delta_{x}$, of $f(x)$ in terms of its samples are as follows:

$$
\begin{aligned}
&\left(\Delta_{\omega}\right)^{2}= \int_{-\pi}^{\pi}|\omega F(\omega)|^{2} \mathrm{~d} \omega \\
&= \frac{1}{2 \pi X}\left[\frac{2 \pi^{3}}{3} \sum_{n \in \mathbb{Z}} f^{2}[n]+\right. \\
&\left.\sum_{m \in \mathbb{Z}} \sum_{\substack{n \in \mathbb{Z} \\
m \neq n}} \frac{4 \pi(-1)^{(m-n)}}{(m-n)^{2}} f[m] f[n]\right] \\
&\left(\Delta_{x}\right)^{2}=\frac{1}{2 \pi} \sum_{m \in \mathbb{Z}} \sum_{n \in \mathbb{Z}} f(m X) f(n X)\left(\frac{m^{2}+n^{2}}{2} X^{2} A+B\right)
\end{aligned}
$$


where

$$
\begin{aligned}
& A=2 W X^{2} \delta_{m n}+ \\
& \frac{X^{2}(-1)^{(m-n)}}{2}\left(\epsilon \frac{\sin ((m-n) X \epsilon)}{(m-n) X \epsilon}+\right. \\
& \left.\frac{\sin ((m-n) X \epsilon)}{2}\left[\frac{2(m-n) X}{\left(\frac{\pi}{\epsilon}\right)^{2}-(m-n)^{2} X^{2}}\right]\right) \\
& +\frac{X^{2}(-1)^{(m-n)}}{2} \\
& \left(\epsilon \frac{\sin ((m-n) X \epsilon)}{(m-n) X \epsilon}\left\{1-\frac{\frac{1}{2}}{\left[1-\left(\frac{(m-n) X \epsilon}{2 \pi}\right)^{2}\right]}\right\}\right)
\end{aligned}
$$$$
B=\frac{1}{4 \epsilon} \frac{\sin ((m-n) X \epsilon)}{(m-n) X \epsilon} \frac{X^{2} \pi^{2}(-1)^{(m-n)}}{\left[1-\left(\frac{(m-n) X \epsilon}{2 \pi}\right)^{2}\right]^{2}}
$$

and $\epsilon$ is a (positive) parameter of the interpolating function appearing in (8), and is less than the difference between $W$ and the actual bandwidth of $f(x)$. When $\epsilon$ tends to 0 , it can be shown, by examining (13), that

$$
A=2 W X^{2} \delta_{m n}
$$

and, from (12),

$$
\begin{gathered}
\left(\Delta_{x}\right)^{2}=\frac{2 W X^{4}}{2 \pi} \sum_{n \in \mathbb{Z}} n^{2} f^{2}(n X)+ \\
\frac{1}{2 \pi} \sum_{m \in \mathbb{Z}} \sum_{n \in \mathbb{Z}} f(m X) f(n X) B
\end{gathered}
$$

And the second term in the above expression involving $B$. when $\epsilon \rightarrow 0$, simplifies to

$$
\begin{array}{r}
\lim _{\epsilon \rightarrow 0} \sum_{m \in \mathbb{Z}} \sum_{n \in \mathbb{Z}} f(m X) f(n X) B= \\
\lim _{\epsilon \rightarrow 0} \frac{1}{4 \epsilon} X^{2}\left|\sum_{n \in \mathbb{Z}} f(n X) \exp (j \pi n)\right|^{2}
\end{array}
$$

Note that if $F( \pm W) \neq 0$, the above expression becomes infinite, when $\epsilon \rightarrow 0$. Assuming that $F( \pm W)=0$, we get

$$
\left(\Delta_{x}\right)^{2}=\frac{2 W X^{4}}{2 \pi} \sum_{n \in \mathbb{Z}} n^{2} f^{2}(n X)
$$

In this special limiting case, the above expression is similar to the one obtained by Ishii [3]. But our procedure is believed to be more general.

In order to make our mathematical analysis more tractable, we analyze finite length discrete time signals. Let $\mathbf{f}=$ $\left(f_{1}, f_{2}, f_{3}, \ldots, f_{N}\right)^{T}$ denote a $N$-dimension column vector representing a discrete sequence. We assume that this finite length discrete sequence is obtained by sampling a bandlimited signal using a sampling interval $X$. Using the samples $\left(\left\{f_{i}\right\}\right)$ of the bandlimited signal, and invoking (1) and (12), we get

$$
\begin{aligned}
& \left(\Delta_{x}\right)^{2}=\mathbf{f}^{T} \mathbf{S} \mathbf{f} \\
& \left(\Delta_{\omega}\right)^{2}=\mathbf{f}^{T} \mathbf{B} \mathbf{f}
\end{aligned}
$$

where $S$ and $B$ are positive definite symmetric, real matrices.

We now find the $\left\{f_{i}\right\}$ which minimizes SBP, $P=$ $\left(\Delta_{x}\right)^{2}\left(\Delta_{\omega}\right)^{2}$, subject to the following two constraints:

1) $\sum_{i}\left(f_{i}\right)^{2}=\frac{1}{X}$; and

2) $F( \pm W)=0$ or, equivalently, $\mathrm{f}^{T} \mathbf{c}=0$ where $c[n]=$ $\exp (j \pi n)$.

Invoking the Lagrange multiplier technique, the above problem reduces to the minimization of modified function, $\mathcal{P}$ :

$$
\mathcal{P}=\left(\mathbf{f}^{T} \mathbf{S} \mathbf{f}\right)\left(\mathbf{f}^{T} \mathbf{B} \mathbf{f}\right)+\lambda\left(\mathbf{f}^{T} \mathbf{f}-1\right)+\mu \mathbf{f}^{T} \mathbf{c}
$$

where $\lambda$ and $\mu$ are Lagrange multipliers. For minimizing the above function, we compute the derivative of $P_{L}$ with respect to $\left(\left\{f_{i}\right\}\right), \lambda$ and $\mu$, and equate them separately to 0 :

$$
\begin{array}{r}
\frac{\partial P_{L}}{\partial \mathbf{f}}=(2 \mathbf{S f})\left(\mathbf{f}^{T} \mathbf{B} \mathbf{f}\right)+(2 \mathbf{B} \mathbf{f})\left(\mathbf{f}^{T} \mathbf{S} \mathbf{f}\right)+2 \lambda \mathbf{f}+\mu \mathbf{c}=0 \\
\frac{\partial P_{L}}{\partial \lambda}=\mathbf{f}^{T} \mathbf{f}-\frac{1}{X}=0 \\
\frac{\partial P_{L}}{\partial \mu}=\mathbf{f}^{T} \mathbf{c}=0
\end{array}
$$

Now we find the values of the Lagrange multipliers $\lambda$ and $\mu$ in terms of $\mathbf{f}$. To this end, we rewrite (21) as

$$
\text { (Sf) }\left(\mathbf{f}^{T} \mathbf{B} \mathbf{f}\right)+(\mathbf{B f})\left(\mathbf{f}^{T} \mathbf{S} \mathbf{f}\right)+\lambda \mathbf{f}+\frac{\mu}{2} \mathbf{c}=\mathbf{0}
$$

Multiplying $\mathbf{f}^{T}$ on both sides of the above equation, we get

$$
\left(\mathbf{f}^{T} \mathbf{S} \mathbf{f}\right)\left(\mathbf{f}^{T} \mathbf{B} \mathbf{f}\right)+\left(\mathbf{f}^{T} \mathbf{B} \mathbf{f}\right)\left(\mathbf{f}^{T} \mathbf{S} \mathbf{f}\right)+\lambda \mathbf{f}^{T} \mathbf{f}+\frac{\mu}{2} \mathbf{f}^{T} \mathbf{c}=0
$$

Using (23) and (22), we can simplify the above equation as

$$
2\left(\mathbf{f}^{T} \mathbf{S} \mathbf{f}\right)\left(\mathbf{f}^{T} \mathbf{B} \mathbf{f}\right)+\frac{\lambda}{X}=0
$$

Finally, we can express $\lambda$ in terms of $f$ as

$$
\lambda=-2 X\left(\mathbf{f}^{T} \mathbf{S} \mathbf{f}\right)\left(\mathbf{f}^{T} \mathbf{B} \mathbf{f}\right)=-2 X \Delta_{x} \Delta_{\omega}
$$

In order to evaluate $\mu$, we multiply $\mathbf{c}^{T}$ on both sides of (24) to get

$$
\begin{array}{r}
\left(\mathbf{c}^{T} \mathbf{S} \mathbf{f}\right)\left(\mathbf{f}^{T} \mathbf{B} \mathbf{f}\right)+\left(\mathbf{c}^{T} \mathbf{B} \mathbf{f}\right)\left(\mathbf{f}^{T} \mathbf{S} \mathbf{f}\right)+\lambda \mathbf{c}^{T} \mathbf{f}+\frac{\mu}{2} \mathbf{c}^{T} \mathbf{c}=0 \\
\left(\mathbf{c}^{T} \mathbf{S} \mathbf{f}\right)\left(\mathbf{f}^{T} \mathbf{B} \mathbf{f}\right)+\left(\mathbf{c}^{T} \mathbf{B} \mathbf{f}\right)\left(\mathbf{f}^{T} \mathbf{S} \mathbf{f}\right)+\frac{\mu}{2} \mathbf{c}^{T} \mathbf{c}=0
\end{array}
$$

Finally, we obtain an expression for $\mu$ in terms of $\mathrm{f}$ as

$$
\begin{aligned}
\mu & =\frac{-2\left[\left(\mathbf{c}^{T} \mathbf{S} \mathbf{f}\right)\left(\mathbf{f}^{T} \mathbf{B} \mathbf{f}\right)+\left(\mathbf{c}^{T} \mathbf{B} \mathbf{f}\right)\left(\mathbf{f}^{T} \mathbf{S} \mathbf{f}\right)\right]}{\mathbf{c}^{T} \mathbf{c}} \\
& =\frac{-2\left[\left(\mathbf{c}^{T} \mathbf{S} \mathbf{f}\right) \Delta_{\omega}+\left(\mathbf{c}^{T} \mathbf{B} \mathbf{f}\right) \Delta_{x}\right]}{\mathbf{c}^{T} \mathbf{c}}
\end{aligned}
$$

Using (24), (27), and (28), we arrive at the following nonlinear equation which the optimal finite length discrete sequence must satisfy: 


\section{CONCLUSIONS}

$$
\begin{array}{r}
(\mathbf{S f})\left(\mathbf{f}^{T} \mathbf{B} \mathbf{f}\right)+(\mathbf{B f})\left(\mathbf{f}^{T} \mathbf{S} \mathbf{f}\right)-2 X\left(\mathbf{f}^{T} \mathbf{S} \mathbf{f}\right)\left(\mathbf{f}^{T} \mathbf{B} \mathbf{f}\right) \mathbf{f} \\
-\frac{\left[\left(\mathbf{c}^{T} \mathbf{S} \mathbf{f}\right)\left(\mathbf{f}^{T} \mathbf{B} \mathbf{f}\right)+\left(\mathbf{c}^{T} \mathbf{B} \mathbf{f}\right)\left(\mathbf{f}^{T} \mathbf{S} \mathbf{f}\right)\right]}{\mathbf{c}^{T} \mathbf{c}} \mathbf{c}=\mathbf{0}
\end{array}
$$

We denote the above vector equation in terms of $\mathbf{f}$ as $\mathrm{L}(\mathbf{f})$ and rewrite it in a simpler form as,

$$
\begin{array}{r}
\mathbf{L}(\mathbf{f})=(\mathbf{S f}) \Delta_{\omega}+(\mathbf{B f}) \Delta_{x}-2 X \Delta_{x} \Delta_{\omega} \mathbf{f} \\
-\frac{\left[\left(\mathbf{c}^{T} \mathbf{S} \mathbf{f}\right) \Delta_{\omega}+\left(\mathbf{c}^{T} \mathbf{B f}\right) \Delta_{x}\right]}{\mathbf{c}^{T} \mathbf{c}} \mathbf{c}=\mathbf{0}
\end{array}
$$

The above equation, is solved by the Newton-Raphson technique by computing the Jacobian matrix of $\mathbf{L}$. For details, see [6]. The results are presented in Figs. 2 and 3. They represent the optimal final length discrete sequences.

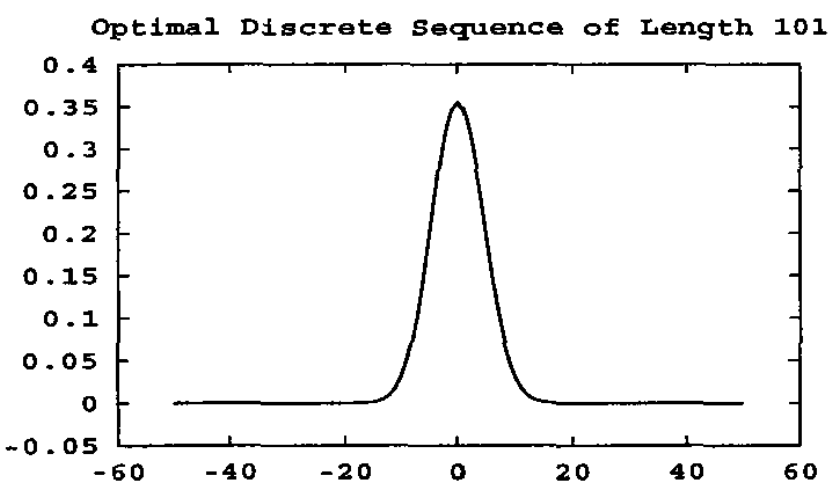

Fig. 2. Optimal bandlimited signal of length 101

Optimal Discrete Sequence of Length 201

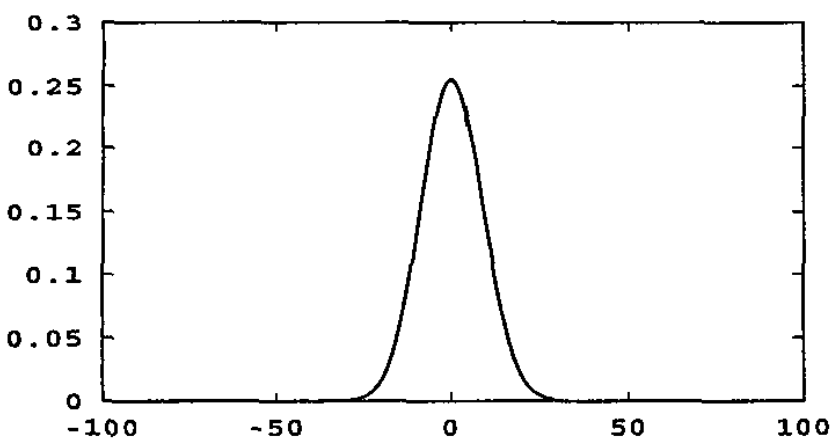

Fig. 3. Optimal bandlimited signal of length 201
In this paper, we have proposed two solutions to the problem of obtaining a bandlimited function $f(x) \in \mathcal{B}$ which has the smallest SBP. The first solution is based on a modification of the Gaussian function; and the second, on the solution of a set of nonlinear algebraic equation obtained from the samples of the signal. It is found that the SBP of any $f(x) \in \mathcal{B}$ is strictly greater than the lower bound as in (4). Further, there are functions in $\mathcal{B}$ whose SBP is very close to the lower bound as in (4).

\section{REFERENCES}

[1] A. Papoulis, Signal Analysis, McGraw-Hill, New York, 1968.

[2] G. B. Folland and A. Sitaram, "The uncertainty principle: A mathematical survey," Journal of Fourier Analysis and Applications, vol. 3, no. 3, pp. 207-238, 1997.

[3] R. Ishii and K. Furukawa, "The uncertainty principle in discrete signals," IEEE Transactions on Circuits Systems, vol. 33, pp. 1032-1034, 1986.

[4] L. C. Calvez and P. Vilbe, "On the uncertainty principle in discrete signals," IEEE Transactions on Circuits Systems II: Analog-Digital Signal Processing, vol. 6, no. 39, pp. 394-395, 1992.

[5] Y. V. Venkatesh, S. Kumar Raja, and G. Vidyasagar, "On the uncertainty inequality as applied to discrete signals," Submitted to IEEE Transactions on Circuits and Systems-II, October 2003.

[6] Y. V. Venkatesh, S. Kumar Raja, and G. Vidyasagar, "On bandlimited signals with minimal space/time-bandwidth product," Technical Report. Department of Electrical Engineering, Indian Institute of Science, Bangalore, December 2003. 